\title{
STRATOSPHERIC OZONE DENSITY RETRIEVAL USING THE OPTIMAL ESTIMATION METHOD (OEM)
}

\author{
Ghazal Farhani $^{1, *}$, R. J. Sica ${ }^{1}$, Sophie Godin-Beekmann ${ }^{2,1}$ and Alexander Haefele ${ }^{3,1}$ \\ ${ }^{1}$ Department of Physics and Astronomy, The University of Western Ontario, Canada \\ *gfarhani@uwo.ca \\ ${ }^{2}$ Université de Versailles Saint-Quentin en Yvelines, France \\ ${ }^{3}$ Federal Office of Meteorology and Climatology MeteoSwiss, Switzerland
}

\begin{abstract}
We use an Optimal Estimation Method (OEM) to retrieve ozone profiles from the CANDAC Stratospheric Ozone Differential Absorption Lidar in Eureka, Canada. The OEM is a well known inverse method in which a forward model (FM) is used to describe the instrument and geophysical situation. We have developed a FM and are testing its validity using synthetic measurements. We will present the advantages of using OEM retrievals over the traditional method, including a full uncertainty budget.
\end{abstract}

\section{INTRODUCTION}

Ozone is a minor constituent in the atmosphere but plays an important role by absorbing harmful ultraviolet (UV) radiation emitted by the Sun. The bulk of the protective ozone resides in the stratosphere (an altitude range of about 12 to $50 \mathrm{~km}$ ) while a small but significant amount exists near the surface, where it is pollutant. Significant chemical depletion of the ozone during late winter and spring has been observed over both Poles [1]. Ozone holes are linked to the emission of chlorine and bromine compounds (the so-called Ozone Depleting Substances or ODS). Since the Montreal Protocol came to effect, the emission of ODSs has been decreased. In order to evaluate the recovery of ozone as a result of this change, continuous measurements of stratospheric ozone is required. Measurements of stratospheric ozone have been carried out using the Differential Absorption Laser technique Lidar (DIAL) in Eu- reka, Nunavut at the Polar Environment Atmospheric Research Laboratory $\left(79.98^{\circ} \mathrm{N}, 85.94^{\circ}\right.$ $\mathrm{W}, 647 \mathrm{~m}$ above sea level) for more than 20 years. In the DIAL method, two different laser wavelengths are simultaneously transmitted to the atmosphere. One wavelength is in a spectral region with a high absorption by ozone (for stratospheric measurements typically $308 \mathrm{~nm}$ ) and is called the "on-line" wavelength. The other wavelength has a low absorption by the ozone (typically $353 \mathrm{~nm}$ ) and is called the "offline" wavelength.

\section{Algorithms to Retrieve Ozone Density for the DIAL System}

The lidar equation gives the intensity of the backscattered photons for a monochromatic laser pulse at wavelength $\lambda$, in an atmospheric layer located at altitude $z$, and acquired during an integration time $\Delta \mathrm{t}$ :

$$
\begin{aligned}
N\left(z, \lambda_{i}\right) & =N_{0}\left(\lambda_{i}\right) \eta\left(\lambda_{i}\right) \frac{A}{z^{2}} \beta\left(\lambda_{i}, z\right) \\
& \times \exp \left[-2 \int_{0}^{z} \alpha\left(z^{\prime}, \lambda_{i}\right) d z^{\prime}\right]
\end{aligned}
$$

where $N_{0}\left(\lambda_{i}\right)$ is the emitted number of photons, $\eta\left(\lambda_{i}\right)$ is the optical efficiency of the receiving system, $A$ is the telescope area, $\beta\left(z, \lambda_{i}\right)$ is the atmospheric backscattering coefficient and $\alpha\left(z, \lambda_{i}\right)$ is the atmospheric extinction coefficient [2]. Traditionally, the ratio of backscattered photons for the two wavelengths is calculated 
and ozone profiles can retrieved as follows:

$$
\begin{aligned}
n o_{3}(z)= & \frac{-1}{2 \Delta \delta_{o 3}(z)} \frac{d}{d z} \ln \left(\frac{N\left(\lambda_{1}, z\right)-N_{b 1}}{N\left(\lambda_{2}, z\right)-N_{b 2}}\right) \\
& +\delta n_{o_{3}}(z)
\end{aligned}
$$

where $N\left(\lambda_{1}, z\right)$ and $N\left(\lambda_{2}, z\right)$ are, respectively, the on-line and off-line signals at altitude $z, N_{b_{2}}$ and $N_{b_{2}}$ are corresponding background signals, $\Delta \delta_{o_{3}}(z)=\delta_{o_{3}}\left(\lambda_{1}\right)-\delta_{o_{3}}\left(\lambda_{2}\right)$ is the differential absorption cross section, and $\delta n_{o 3}(z)$ is a correction term. The detailed calculation is presented in [3] and [4].

The Optimal Estimation Method (OEM) is a well known method in the passive remote sensing method; however, it has only been recently used to retrieve atmospheric quantities with lidar measurements (for example [5]). The OEM has many advantages such as providing a full uncertainty budget, including both systematic and statistics errors of the retrieved profiles. In the OEM method, (unlike the traditional method) no pre- or post- filtering of retrievals is required (often digital filters will introduce uncertainties to the final result [3]). Moreover, the sky background correction can be retrieved along wihh the ozone density profile. A brief introduction of the method is covered in the next section. Comprehensive details on the subject can be found in [6].

\section{METHODOLOGY}

The Optimal Estimation Method (OEM) is a matrix inverse method based on Bayes' theorem. Let $\mathbf{x}=\left(x_{1}, x_{2}, \ldots, x_{n}\right)$ be the state vector of the atmosphere and $\mathbf{y}=\left(y_{1}, y_{2}, \ldots, y_{n}\right)$ be the corresponding measurement vector. The relationship between $\mathbf{x}$ and $\mathbf{y}$ is called the forward model (FM):

$$
\mathbf{y}=F(\mathbf{x}, b)+\varepsilon
$$

where $b$ is the model parameter vector and $\varepsilon$ is the measurement error vector. For non linear problems, the FM can be linearized:

$$
\begin{array}{r}
y-F\left(x_{a}\right)=\left.\frac{\partial F}{\partial y}\right|_{x_{a}}\left(x-x_{a}\right) \\
+O\left(x^{2}\right) \approx K\left(x-x_{a}\right)
\end{array}
$$

where $\mathbf{K}_{x}=\frac{\partial F}{\partial y}$ is the Jacobian matrix and represents the sensitivity of the observation variables $\mathbf{y}$ to the state variables $\mathbf{x}$ [6]. The $\mathbf{K}$ represents a linearization of the forward model around $x$. It initially needs to be calculated for the a priori $x_{a}$, and then recalculated for the updated values of $x$ during the iterative convergences to the solution. The iteration continues to minimize the cost function $J(x)$ :

$$
\begin{array}{r}
J(X)=[y-F(\widehat{x}, b)]^{T} S_{y}^{-1}[y-F(\widehat{x}, b)] \\
+[x-\widehat{x}]^{T} S_{a}^{-1}[x-\widehat{x}] .
\end{array}
$$

The solution is:

$$
\widehat{x}=x_{a}+S_{a} K^{T}\left(K S_{a} K^{T}+S_{a}\right)^{-1}\left(y-K x_{a}\right)
$$

where $\mathbf{S}_{a}$ and $\mathbf{S}_{\varepsilon}$ are the uncertainties associated with an a priori state and measurements, their diagonal elements are the variances of the individual elements of the a priori state and measurements error.

\subsection{Applying the OEM for Ozone Profile Retrieval}

Using two uncorrelated measurements we are producing first ozone retrievals. For the pilot study, we are testing the OEM on synthetic measurements. After being confident about the results, we will apply our method to real measurements. Determining the appropriate FM is one of the main challenges in the OEM. We are using the lidar equation as our FM.

$$
\begin{array}{r}
N\left(z, \lambda_{i}\right)=N_{0}\left(\lambda_{i}\right) \eta\left(\lambda_{i}\right) \frac{A}{z^{2}} \beta\left(\lambda_{i}, z\right) \\
\quad \times \exp \left[-2 \int_{0}^{z} \alpha\left(z^{\prime}, \lambda_{i}\right) d z^{\prime}\right]+B .
\end{array}
$$

Here, $N\left(z, \lambda_{i}\right)$ represents the detected photons (without applying any correction). In the DIAL method two wavelengths are simultaneously 

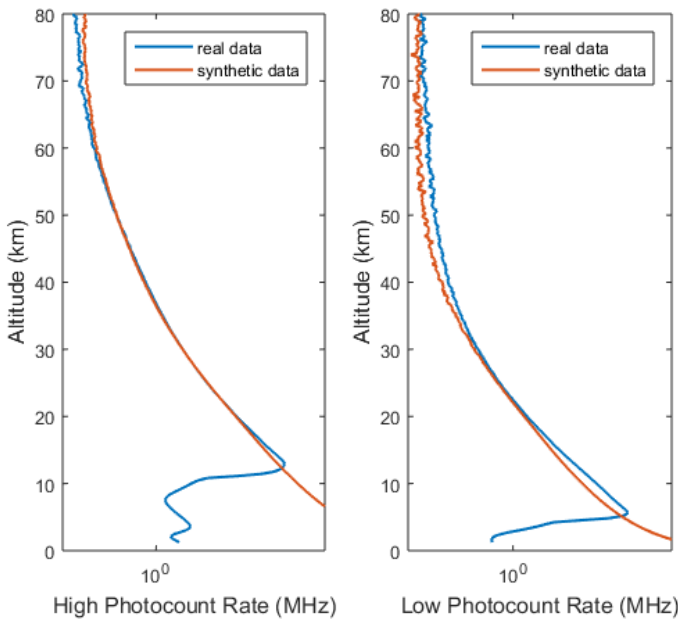

Figure 1: Synthetic countrate for 3 hours of DIAL measurements on Feb. 28, 2016. Left panel: high channel (blue curve, real measurements; red curve, synthetic measurements). Right panel: low channel.

transmitted. We only use the on-line wavelength for our calculations for this initial study, but are currently extending the retrieval to use both data channels.

\section{RESULTS}

Using the US standard atmospheric model ozone profile we made our synthetic measurements for both high and low Rayleigh channels at $308 \mathrm{~nm}$ (see Figure 1). These profiles are similar to 3 hours of the Eureka DIAL measurements on 28 of February 2016. We assumed a constant background in generating our synthetic measurements. The assumption provides a good fit for the low channel counts. However, the background count for the high channel above $60 \mathrm{~km}$ due to the signal induced noise (SIN) effect is not a constant value. We plan to include the effect of SIN offset on the background counts in the near future.

We used the same ozone profile for our a priori, therefore, we expect to get the optimal solution without any iteration. The averaging kernel shows the sensitivity of the retrieval to the vector state. As shown in Figure 2, the averaging kernel equals 1 below $40 \mathrm{~km}$ which means the retrieved profile below this altitude is inde- pendent of the a priori information. The sensitivity of the averaging kernel decreases with increasing altitude.

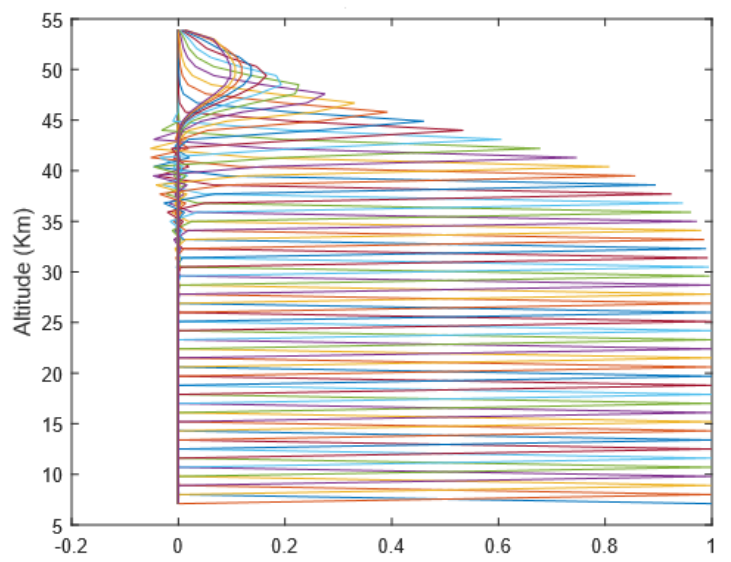

Figure 2: Averaging kernel for the ozone profile for the measurements shown in Fig.1. The averaging kernel equals 1 below $40 \mathrm{~km}$.

The residuals (see Figure 3) show the difference between the FM and the measurements (here the synthetic data). The residuals (shown in blue) are within the FM uncertainty (shown in red).
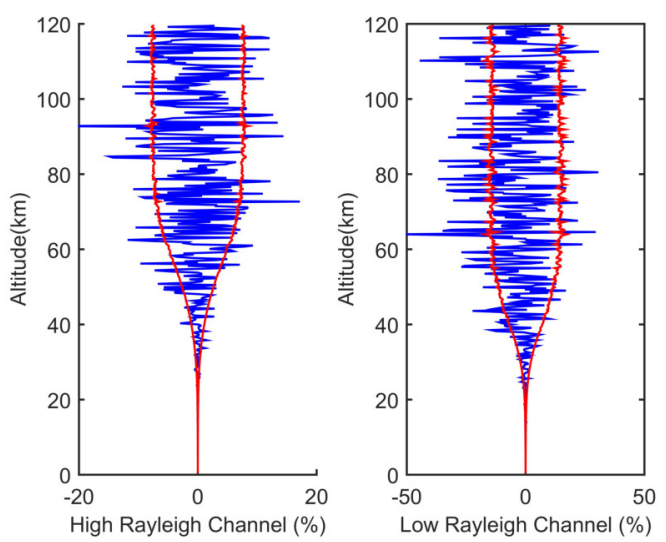

Figure 3: Residuals between the forward model and the measurements for the low and high channels (blue). The red lines show the standard deviation of the measurements.

Figure 4 shows the retrieved ozone profile. The retrieval grid is extended from $7 \mathrm{~km}$ to $50 \mathrm{~km}$. The retrieved ozone profile tends to fall back 
to the a priori ozone profile at altitudes above $40 \mathrm{~km}$ which is predictable as the sensitivity of the averaging kernel is decreasing above this height. The cost of this retrieval is 1.03.

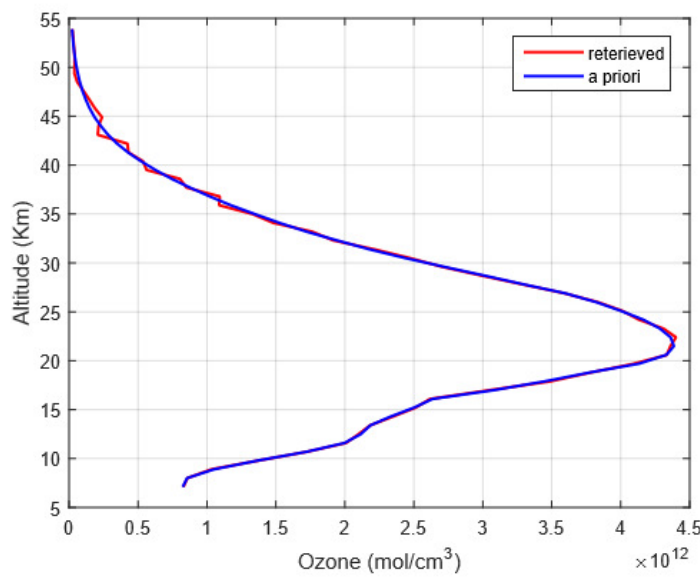

Figure 4: Retrieved ozone profile (red curve) using the OEM.The blue curve is the a priori ozone profile used by the OEM.

The difference between the retrieved profile and the $a$ priori profile is shown in Figure 5.

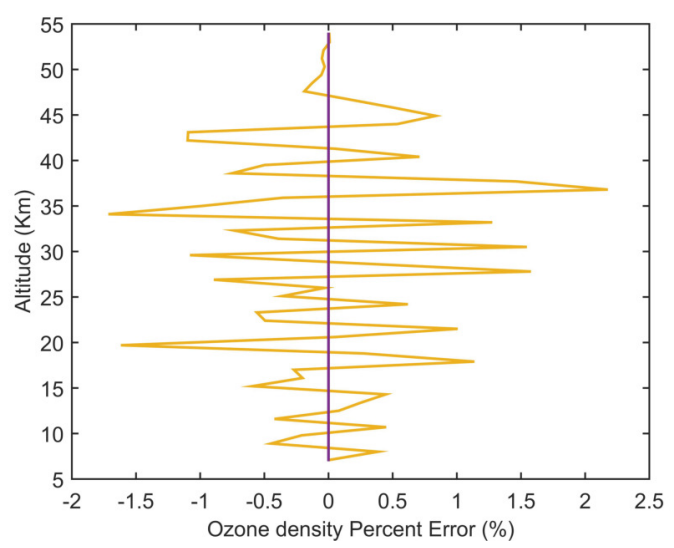

Figure 5: The percentage error between the retrieved ozone profile and the a priori ozone profile.

\section{CONCLUSIONS}

We use an Optimal Estimation Method (OEM) to retrieve ozone profiles from synthetic measurements from $7 \mathrm{~km}$ to $50 \mathrm{~km}$. The retrieval is currently being extended to include background counts and the lidar constants using two data channels. We are also planning to retrieve the aerosol extinction coefficient. We also are analysing real measurements and are validating the retrievals against other measurements.

\section{ACKNOWLEDGEMENTS}

We thank the Natural Science and Engineering Research Council (NSERC), the Canadian Space Agency (CSA) and, the Northern Scientific Training Program (NSTP).

\section{References}

[1] S. Solomon. Stratospheric ozone depletion: A review of concepts and history. Reviews of Geophysics, 37(3):275-316, 1999.

[2] R. M. Measures. Laser Remote Sensing: Fundamentals and Applications. Krieger Pub Co; New edition edition (August 1991).

[3] S. Godin, A. Carswell, D .Donovan, H. Claude, W. Steinbrecht, S. McDermid, T. McGee, M. Gross, H. Nakane, D. Swart, et al. Ozone differential absorption lidar algorithm intercomparison. Applied optics, 38(30):62256236, 1999.

[4] T. Leblanc and F. Gabarrot. Critical assessment and standardized reporting of vertical filtering and error propagation in the data processing algorithms of the ndacc lidars. Technical report, NDAAC, 2015.

[5] R. J. Sica and A. Haefele. Retrieval of temperature from a multiple-channel rayleigh-scatter lidar using an optimal estimation method. volume 54, pages 1872-1889. OSA, Mar 2015.

[6] C. D. Rodgers. Inverse Methods for Atmospheric Sounding: Theory and Practice. World Scientific Publishing, 2000. 